\title{
An Enhanced Distance Vector-Hop Algorithm using New Weighted Location Method for Wireless Sensor Networks
}

\author{
Fengrong $\operatorname{Han}^{1 *}$, Izzeldin Ibrahim Mohamed Abdelaziz ${ }^{2}$, Xinni Liu ${ }^{3}$, Kamarul Hawari Ghazali ${ }^{4}$ \\ Faculty of Electrical \& Electronics Engineering, \\ Universiti Malaysia Pahang \\ Pahang, Malaysia
}

\begin{abstract}
Location is an indispensable segment for Wireless Sensor Network (WSN), since when events happened, we need to know location. The distance vector-hop (DV-Hop) technique is a popular range-free localization algorithm due to its cost efficiency and non-intricate process. Nevertheless, it suffers from poor accuracy, and it is highly influenced by network topology; Especially, more hop counts lead to more errors. In the final phase, least squares are employed to address nonlinear equation, which will gain greater location errors. Aimed at addressing problems mentioned above, an enhanced DV-Hop algorithm based on weighted factor, along with new weighted least squares location technique, is proposed in this paper, and it is called WND-DV-Hop. First, the one hop count of unknown node was corrected by employed received signal strength indication (RSSI) technology. Next, in order to reduce average hop distance error, a weighted coefficient based on beacon node hop count was constructed. A new weighted least squares method was embedded to solve nonlinear equation problem. Finally, considerable experiments were carried out to estimate the performance of WND-DV-Hop, compared the outcomes with state-of-the-art DV-Hop, IDV-Hop, Checkout-DV-Hop, and NewDV-Hop depicted in literature. The empirical findings demonstrated that WND-DV-Hop significantly outperformed other localization algorithms.
\end{abstract}

Keywords-Wireless Sensor Network (WSN); localization algorithm; range-free; distance vector-hop (DV-Hop) localization algorithm

\section{INTRODUCTION}

Advanced electronics and cutting-edge wireless communication technologies have fostered large-scale wireless sensor network (WSN). The WSN is composed of great amounts of self-organizing, tiny size, and limited computational sensor nodes, which reflects a multiple hop network [1,2]. The WSN has been considered as one of the most promising technologies to deal with tough issues. It has been successfully employed in disparate areas for monitoring and tracking purposes, such as military, public industry, agriculture, environment, and health care [3, 4].

Location is a fundamental issue in WSN, as it is crucial to identify where the information is derived from, mainly because the data becomes meaningless with information about location. This is especially true for real-time applications that demand precise location-based services [5]. Some fundamental techniques developed for WSN require sensor location information, such as geographical routing protocols [6]. Additionally, several principle location-based network services need support from accurate location data, such as network coverage optimization, topology structure, and beacon node clustering [7].

Exact locations may be picked from sensor nodes attached to Global Positioning System (GPS) chips. Yet, this incontrovertibly increases hardware costs despite being equipped as a part of the nodes. The performance of localization accuracy, nonetheless, is not good if GPS is installed in tall buildings or other unsuitable environments surrounded by obstacles. Many scholars have proposed a range of algorithms and models. The localization schemes could be broadly grouped into range-free and range-based localization [5], depending if they need to attach additional hardware device(s). The range-free algorithms only utilize network connectivity data and hop information to calculate the location of the sensor nodes, thus easy to apply and operate. Some of these classic algorithms are Amorphous [8], Centroid [9], Distance Vector-Hop (DV-Hop) [10] and Approximate Point in Triangle Test (APIT) [11]. The range-based localization techniques embed various measuring techniques to collect favorable location data, including Received Signal Strength Indicator (RSSI) [12, Time of Arrival (ToA) [13], Time Difference of Arrival (TDoA) [13], and Angle of Arrival (AoA) [14]. These techniques offer higher localization accuracy with extra hardware cost. Nonetheless, they are sensitive to environmental noises and easily influenced by barriers. Hence, rang-free localization algorithm became to most popular location method.

The DV-Hop propagation model has attracted much attention from researchers worldwide for its advantages of simplicity, feasibility, cost-efficiency, and high coverage. It does not rely on measurement error, easy to understand and implement, and has broad popularity within the localization domain. As such, this study investigated the DV-Hop scheme.

The essential function of DV-Hop is to calculate nodes distances by multiplying average hop size and hop counts between nodes. Suppose that the path of minimum hop is nearly beeline, the initial DV-hop algorithm performs better, exclusively when the distribution of nodes is uniform. If otherwise, a wide gap can be expected between the calculated 
and the actual location. The literature presents a sea of related approaches to address above issues. Chen et al. [15] proposed a rapid, accurate, and easy DV-Hop localization algorithm called IDVLA. It computes the average of the entire hop size, instead of the initial hop. After that, a new weighted least squares method was used to substitute least squares. Next, an enhanced algorithm, hybrid DV-Hop algorithm, was initiated by Omar et al. [16]. If hop count between unknown and beacon node is 1 , the RSSI method was used to estimate distances, or otherwise, the conventional method was applied. Upon localization, the node serves as an assistant beacon node. According to Peng et al. [17], although the GADV-Hop algorithm used genetic algorithm (GA) to minimize the total estimation error, it suffers from high computation complexity. Thus, a trade-off was embedded between localization accuracy and computation intricacy for a new proposed scheme. A comprehensive review of previous work is presented in Section 2.

This study proposes an enhanced DV-Hop localization algorithm based on weighted factor along with a new weighted least squares method for WSN.

The primary contributions of this paper are summarized in the following:

1) One hop node and its estimated distances is corrected by using the RSSI technique.

2) The average hop size is calibrated by a weighted coefficient to minimize the estimated distance error.

3) Finally, a new weighted least square is proposed to address the nonlinear equation to estimate node coordinates.

The remaining segment of this paper is as follows. Newborn literature pertaining to DV-Hop is reviewed in Section 2. In Section 3, error analysis of basic DV-Hop is described. Elaboration of proposed WND-DV-Hop algorithm is depicted in Section 4. In Section 5, simulation outcomes and performance evaluation are discussed. Finally, conclusions and future work are presented in Section 6.

\section{RELATED WORKS}

This section presents several remarkable studies concerned DV-Hop algorithm. Details pertaining to localization algorithm process are given in the following. Gui et al. [18] proposed two new algorithms based on improved protocol. They merely applied three nearest beacons for unknown node, instead of using all connected beacons to detect the location. It was assumed that two sensor nodes had consistent connectivity and close position. The hypothesis, however, is not always satisfied due to random deployment. Tomic et al. [19] initiated three new localization proposals. The first two proposals applied the geometry method to determine the best beacon node, while the third algorithm substituted it as bounded least squares issue, wherein quadratic programming was used to address. The simulation results proved that the three algorithms gave better performances, but increased computational complexity. A new approach of weighted hop distance is presented in [20]. The average size distance was reduced based on the weighted coefficient value. The simulation outcomes showed improvement in localization accuracy by $10 \%-15 \%$. To enhance the accuracy, a new localization algorithm was investigated in [21]. First, the estimated distance error was modified by using the orthogonal polynomial fitting approach in the second stage. Next, square after subtraction was employed in the third stage. Finally, a weighted matrix is used to refine the coordinates of unknown node.

Song et al. [22] introduced two refined algorithms. The first used the mean value of all average hop size, instead of the conventional one that decreased the error by $15 \%-20 \%$. The IWC-DV-hop algorithm reduced the error by $9 \%$, which enhanced the accuracy by selecting suitable beacon nodes combined with centroid localization. Zhang et al. [23], first, analyzed drawbacks of DV-hop. Next, they proposed a new weighted localization combined with centroid algorithm, which improved the accuracy by $10 \%$. It was concluded that the nearest beacon had a higher impact. Fang et al. [24] presented a compensation coefficient to revise the estimated distances, which could be applied for both random distribution and dynamic topology networks. The simulation results demonstrated that it gave better performance in location accuracy, and reduced error by $18 \%$. However, the proposed algorithm increased both computation overhead and computation time.

In [25], DV-MaxHop was proposed for anisotropic network. Only maximum hop count beacon was selected for location estimation decision, while the rest were omitted. By selecting the optimal MaxHop, it achieved enhancement by $20 \%$ in terms of accuracy and efficiency. Wang et al. [26] proposed a hybrid GA with simplex method to gain better accuracy. The weighted coefficient was employed to calculate the estimated distance. Finally, GA combined with simplex method was introduced. Kumar et al. [27] proposed NDV-Hop localization that revised the hop size of unknown nodes by introducing a boost term that greatly eliminated communication. Next, they applied the unconstrained optimization technique to minimize error terms, which significantly minimized time and energy consumption. The simulation results demonstrated that the location error was lower by $18 \%$.

Kaur et al. [28] proposed a nature-inspired algorithm, GWO-DV-Hop. First, Grey-Wolf optimization was employed to enhance hop size by combining grey wolf optimizer. Next, a weighted Grey-Wolf was applied to the weighted average hop distance by considering the impact of all beacons. The proposed algorithm improved the localization accuracy by almost $10 \%$. Kaur et al. [29] also presented a new localization using single mobile beacon node based on advanced path model. It displayed higher accuracy and network coverage, despite under sparse network. Kaur et al. [30] also introduced the Gauss-Newton method to address nonlinear method. This algorithm was implemented under 3D WSN and had managed to reduce error by almost 20\%. In [31], three improved DVhop localization algorithms based on optimization techniques are presented. The average localization errors of the three algorithms had been declined. The ICA-DV-hop resulted in the highest localization accuracy among the three algorithms. Deepak et al. [32] introduced a new metric-based method, in which the author proved that it gave less errors, when compared to the conventional DV-Hop using mathematical analysis. 
All proposed algorithms have enhanced localization accuracy to a certain extent, as most of them have several shortcomings, including computational complexity and communication overhead. Hence, this study had attempted to enhance localization accuracy, minimize communication overhead, and reduce computation intricacy.

\section{DV-HOP AND RSSI LOCALIZATION ALGORITHM}

\section{A. DV-Hop Localization Algorithm}

DV-Hop localization scheme was designed by Dragons Niculescu and his team [10] for 2D WSN, which was a classic range-free distributed localization algorithm. Generally, it was divided into three parts.

\section{Phase 1: Calculate Minimum Hop Counts}

In phase 1 , the beacon node, $A_{i}$, broadcasts a packet $\left\{\right.$ ID; $x_{i}$, $\left.y_{i}, h i\right\},\left(x_{i}, y_{i},\right)$ that represents the coordinated of $A_{i}, h_{i}$ is hop count, which is increased by 1 from its initial value of 0 . If node $B$ receives a smaller hop count, it will update $H_{i}$, otherwise, the packet will be discarded. Every node gets its minimum hop counts to all beacon nodes by this mechanism.

\section{Phase 2: Calculate of Average Hop Size}

In the second phase, beacon node, $A_{i}$, calculates its average hop size, expressed as $\mathrm{AvgHopSize}_{i}$ using Equation (1) given in the following:

$\operatorname{AvgHopSize}_{i}=\frac{\sum_{i \neq j}^{m} \sqrt{\left(x_{i}-x_{j}\right)^{2}+\left(y_{i}-y_{j}\right)^{2}}}{\sum_{i \neq j}^{m} H_{i j}}$

Where, $\left(x_{i}, y_{i}\right)$ and $\left(x_{j}, y_{j}\right)$ are the coordinates of beacon nodes $i$ and $j$, respectively. Meanwhile, $H_{i j}$ is the minimum hop-count value between them, and $\mathrm{m}$ is the number of beacon nodes.

After received the hop size, each beacon node broadcasts its AvgHopSize $_{i}$ in the network. The unknown node can calculate distance, $d_{i u}$, from the nearest beacon node by multiplying AvgHopSize $e_{i}$ with hop count using Equation (2) given in the following:

$d_{i u}=$ AvgHopSize $_{i} \times H_{\text {iu }}$

Where $d_{i u}$ and $H_{i u}$ are estimate distance and the minimum hop count between beacon node $i$ and unknown node $u$, respectively.

\section{Phase 3: Calculation of Unknown Nodes Coordinates}

In the last phase, the coordinate of each located node is calculated by using least squares or maximum likelihood estimation method.

It is assumed that $\left(x_{u}, y_{u}\right)$ are the coordinates of unknown node $u$, and let $d_{i u}$ be distance between $u$ and $A_{i}, i \in\{1,2$, $3 \ldots n\}$, where $n$ is the number of communicable beacon nodes. Equation (3) is expressed as follows:

$$
\begin{gathered}
\left(x_{u}-x_{1}\right)^{2}+\left(y_{u}-y_{1}\right)^{2}=d_{1 u}^{2} \\
\left(x_{u}-x_{2}\right)^{2}+\left(y_{u}-y_{2}\right)^{2}=d_{2 u}^{2} \\
\cdot \\
\cdot \\
\left(x_{u}-x_{n}\right)^{2}+\left(y_{u}-y_{n}\right)^{2}=d_{n u}^{2}
\end{gathered}
$$

Each equation was subtracted from the last equation since the first one, Equation (3), can be expressed as:

$$
\begin{aligned}
2\left(x_{n}-x_{1}\right) x_{u}+2\left(y_{n}-y_{1}\right) y_{u} & =d_{1}^{2}-d_{n}^{2}-x_{1}^{2}+x_{n}^{2}-y_{1}^{2}+y_{n}^{2} \\
2\left(x_{n}-x_{2}\right) x_{u}+2\left(y_{n}-y_{2}\right) y_{u} & =d_{2}^{2}-d_{n}^{2}-x_{2}^{2}+x_{n}^{2}-y_{2}^{2}+y_{n}^{2} \\
& \cdot \\
2\left(x_{n-1}-x_{n}\right) x_{u}+2\left(y_{n-1}-y_{n}\right) y_{u} & =d_{n-1}^{2}-d_{n}^{2}-x_{n-1}^{2}+x_{n}^{2}-y_{n-1}^{2}+y_{n}^{2}
\end{aligned}
$$

Equation (4) can be transformed into $\mathrm{AX}=\mathrm{B}$;

$A=-2 \times\left[\begin{array}{cc}x_{1}-x_{n} & y_{1}-y_{n} \\ x_{2}-x_{n} & y_{2}-y_{n} \\ & \cdot \\ & \cdot \\ x_{n-1}-x_{n} & y_{n-1}-y_{n}\end{array}\right]$

$B=\left[\begin{array}{c}d_{1}^{2}-d_{n}^{2}-x_{1}^{2}+x_{n}^{2}-y_{1}^{2}+y_{n}^{2} \\ d_{2}^{2}-d_{n}^{2}-x_{2}^{2}+x_{n}^{2}-y_{2}^{2}+y_{n}^{2} \\ \cdot \\ \cdot \\ d_{n-1}^{2}-d_{n}^{2}-x_{n-1}^{2}+x_{n}^{2}-y_{n-1}^{2}+y_{n}^{2}\end{array}\right]$

$X=\left[\begin{array}{l}x_{u} \\ y_{u}\end{array}\right]$

The unknown node $\left(x_{u}, y_{u}\right)$ can obtain its estimated coordinate based on least square estimations or maximum likelihood estimation method, as follows:

$X=\left(A^{T} A\right)^{-1} A^{T} B$

\section{B. RSSI Localization Algorithm}

The RSSI was proposed by L. Girod et al. in [8]. The estimate distance between sender and receiver can be obtained by using received power strength under a specific path loss model, as given in Equation (9).

$p_{r}(d)=p_{r}\left(d_{0}\right)+10 n \log _{10} \frac{d}{d_{0}}+X_{\sigma}$

Where, $d$ is the distance between sender and receiver nodes, $p_{r}(d)$ denotes received signal strength at distance $d, d_{0}$ represents the reference distance, usually taken as $1 \mathrm{~m}$, and $n$ is the path loss exponent. $X_{\sigma}$ refers to power loss due to the shadowing effect on actual environments.

Let $d_{0}=1, R S S I=p_{s}-p_{r}(d), A=p_{s}-p_{r}\left(d_{0}\right)$, RSSI can be simplified into Equation (10).

$R S S I=A-10 n \log _{10} \frac{d}{d_{0}}+X_{\sigma}$

Next, estimated distance, $d$, can be determined from Equation (11).

$d=10 \frac{A-R S S I}{10 n}$ 


\section{Error Analysis for Basic DV-Hop Algorithm}

- Error by Minimum Hop Count

Problem 1:

The DV-Hop estimates its hop size by hop counts and distances between sensor nodes. Hop-count is discontinuous as the nodes are irregularly deployed within the monitoring region. This suggests a rather major error since several nodes may share similar hop-count with identical beacon node.

In Fig. 1, the red and blue keys denote beacon and unknown nodes, respectively. Fig. 1 depicts that unknown nodes $j, k$, and $m$ are within the communication radius of $\mathrm{R}$, whereby the three of them are one-hop node to beacon node $i$. Consequently, the estimated distance between beacon node $i$ and unknown nodes $j, k$, and $m$ is same. Nonetheless, the actual distance between them is apparently different. The estimation scheme of DV-Hop, thus, would lead to a blatant error. This erred trend has motivated this study to formulate an advanced approach that estimates precise minimum hop.

- Accumulated Estimated Error by Average Hop Size

Problem 2:

The basic DV-Hop presumes the minimum hop is nearly beeline distribution, which is in contradictory in the actual scenario. This is especially true when the sensor nodes in WSN are in spares, hence disabling the average hop size to represent the whole network. This demands a modification to be made on the average hop size.

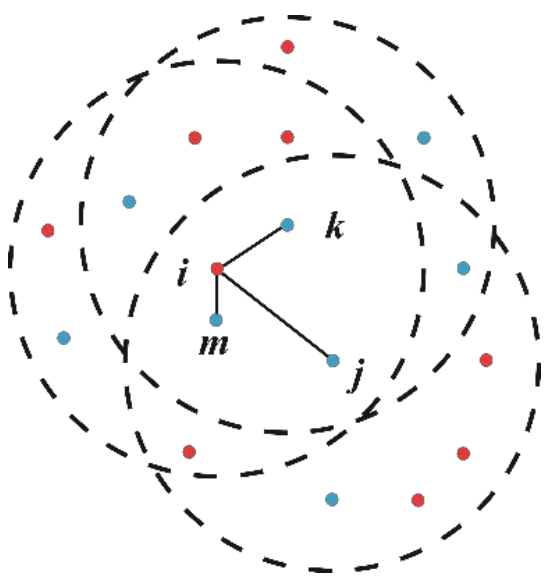

Fig 1. The Relationship of Node Distribution.

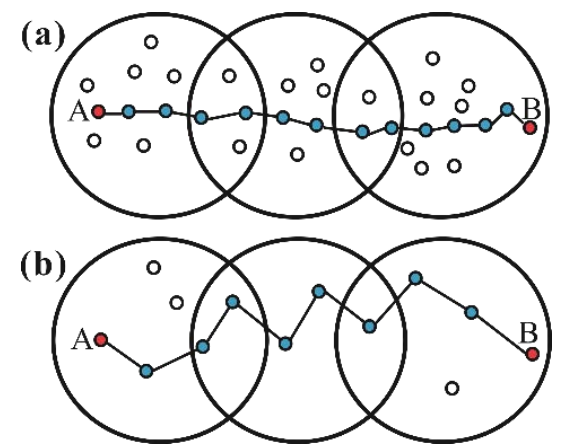

Fig 2. Node Distribution Model; (a), this is a Case Model Node Distribution in Dense; (b), this is a Case in Spares.
Based on Fig. 2(a), since the distance between A and B is on the same line with high network density, the estimated distance is accurate. Nevertheless, in Fig. 2(b), the average hop distance differs, thus resulting in a massive error of estimate distance between A and B to the actual distance. Besides, in phase 3, the unknown node is bound to select the nearest beacon node's average hop size to be multiplied with hop count, hence leading to a grave error.

\section{Proposed Algorithm WND-DV-HoP}

\section{A. Improved DV-Hop based on Weighted Correction}

Phase 1: The conventional DV Hop algorithm was used in this phase with each node having a minimum hop count.

Phase 2: In this phase, the conventional algorithm was improved to determine the correction of one hop count of the unknown node. The average hop size was revised in this phase.

- Correct One Hop Count to Unknown Node

This sub-section describes the improved algorithm to address problem 1 (see Section III, C).

If the hop count of unknown node to neighbour beacon node is 1 , the estimated distance is determined using RSSI Equation (11) instead of Equation (2). This enables a comparison of the received wireless signal strength with beacon node at $(\mathrm{R} / 2)$. If $p_{r}(d)$ exceeds $p_{r}(R / 2)$, its hop value is 0 . 5 , while 1 hop if otherwise. This method introduces the hop hierarchical processing on nodes whose hop is 1 , so that the hop value is no longer an integer. This discretisation process can effectively enhance the credibility of hop counts.

\section{- Correct Average Hop-size of Beacon Nodes} C).

This part discusses to overcome problem 2 (see Section III,

Beacon and unknown nodes are denoted as $m$ and $n$, respectively. Let coordinates of beacon nodes $I$ and $J$ be $\left(x_{i}, y_{i}\right)$ and $\left(x_{j}, y_{j}\right)$, respectively. The average hop size of beacon node $I$ can be determined using Equation (1). The average of $\mathrm{AvgHopSize}_{i}$ is calculated using Equation (12):

$\overline{\text { AvgHopSize }}=\sum_{i=1}^{m}$ AvgHopSize $_{i}$

Here, $\overline{A v g H o p S i z e}$ is employed instead of AvgHopSize ${ }_{i}$. The estimated distance between beacon nodes $I$ and $J$ can be estimated using Equation (13):

$d_{i j}^{e s t}=\overline{\text { AvgHopSize }} \times H_{i j}$

Where, $H_{i j}$ refers to hop count between beacon nodes $I$ and $J$. Hence, the actual distance between them is as follows:

$d_{i j}^{a c t}=\sqrt{\left(x_{i}-x_{j}\right)^{2}+\left(y_{i}-y_{j}\right)^{2}}$

Hop error is given in the following: 
$E_{i}=\frac{1}{m-1} \sum_{\substack{i=1 \\ i \neq j}}^{m} \frac{\left(d_{i j}^{a c t}-d_{i j}^{e s t}\right)}{H_{i j}}$

The new average of hop size is given below:

AvgHopSize $_{i}^{\text {new }}=$ AvgHopSize $_{i}-E_{i}$

\section{- Correct Estimate Distance of Unknown Node}

\section{1) One Hop}

If the hop between unknown and beacon nodes is 1 , the distance is estimated determined using RSSI approach with Equation (11), instead of Equation (2).

\section{2) Multiple Hop Counts}

The weighted coefficient was employed to correct the estimated distance for multiple hop counts. The details are given in the following.

If both beacon and unknown nodes are closer, the average error per hop becomes smaller. This offers a more accurate estimated distance of the unknown node hop. Information regarding the location of beacon node obtained by unknown node $U$ is $k$. The unknown node assigns varying weights to each beacon node (e.g. beacon node $I$ ). Here, $W_{i}$ is computed using the following equation:

$w_{i}=\frac{\frac{1}{E_{i}}+\frac{1}{H_{i u}}}{\sum_{i=1}^{k}\left(\frac{1}{E_{i}}+\frac{1}{H_{i u}}\right)}$

Based on the above calculation, the distance between unknown node $U$ and beacon node (e.g. $I$ ) can be obtained by the following:

$d_{u i}=\left(\sum_{i=1}^{k}\left(w_{i} \times\right.\right.$ AvgHopSize $\left.\left._{i}^{\text {new }}\right)\right) \times H_{u i}$

Phase 3:

Yan et al. [33] proposed an optimal weighted least square for irregular network. Based on this idea, we introduced it to regular network, employed a weighted coefficient matrix $\mathrm{W}$ to address larger error caused by least squares method, see as following.

$W=\left[\begin{array}{cccc}W_{1} & 0 & 0 & 0 \\ 0 & W_{2} & 0 & 0 \\ . & . & . & . \\ . & . & . & . \\ 0 & 0 & 0 & W_{k}\end{array}\right]$

Where, $W_{k}=1 / H_{k}^{3}, H_{k}^{3}$ is the minimum number hop count between target node $X$ and anchor node $A$. Hence, Equation (8) can be transformed into Equation (20).

$X=\left(A^{T} W^{T} W A\right)^{-1} A^{T} W^{T} W B$

\section{B. Steps of Improved Algorithm WND-DV-Hop}

Step 1. Minimum hop count is acquired by flooding protocol among nodes, which is like that for DV-Hop localization algorithm in phase 1.

Step2. Each node knows its shortest hop count. If the hop count of unknown nodes to neighbour beacon node is 1 , RSSI is employed to correct its hop count and to estimate its distance to neighbour beacon node. The information table is updated and the message is forwarded to their neighbours.

Step 3. This phase is like basic DV-Hop, whereby each AvgHopSize of beacon node is calculated by Equation (16) to correct the average hop size.

Step 4. The weighted coefficient is applied to correct the estimate distance of unknown node with multiple hop counts.

Step 5. The coordinates of unknown nodes are calculated by weighted least square method. The flow diagram of improved algorithm (WND-DV-Hop) is illustrated in Fig. 3.

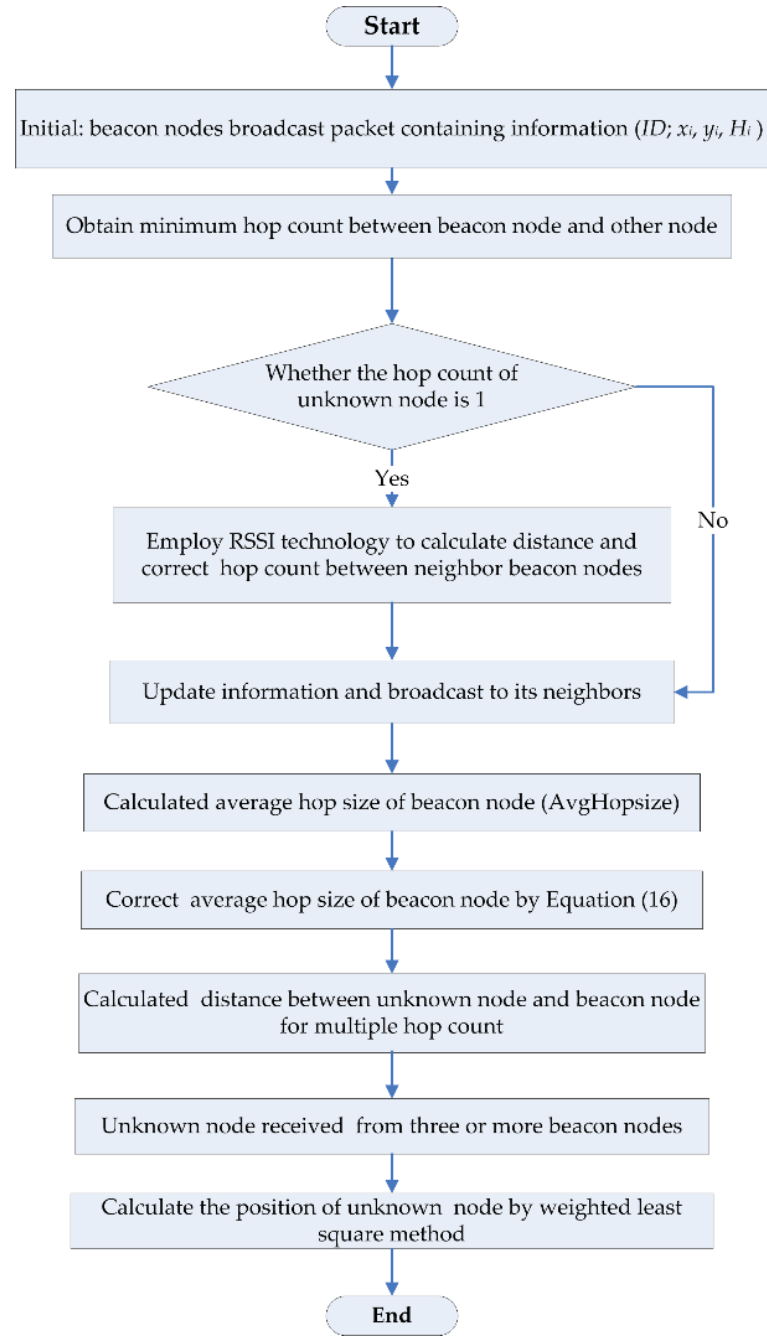

Fig 3. The Flowchart of our Proposed Algorithm (WND-DV-Hop). 


\section{EXPERIMENTAL RESULTS AND ANALYSIS}

The performance of the proposed WND-DV-Hop algorithm had been assessed by weighing in localization accuracy, localization stability, and computational cost under several conditions, such as node density, beacon node ratio, and communication radius. A classic representation of node distribution in 2D space is portrayed in Fig. 4. A total of 150 nodes are randomly displayed in the $150 \times 150 \mathrm{~m}^{2}$ area, including 30 beacon nodes denoted by red pentacles.

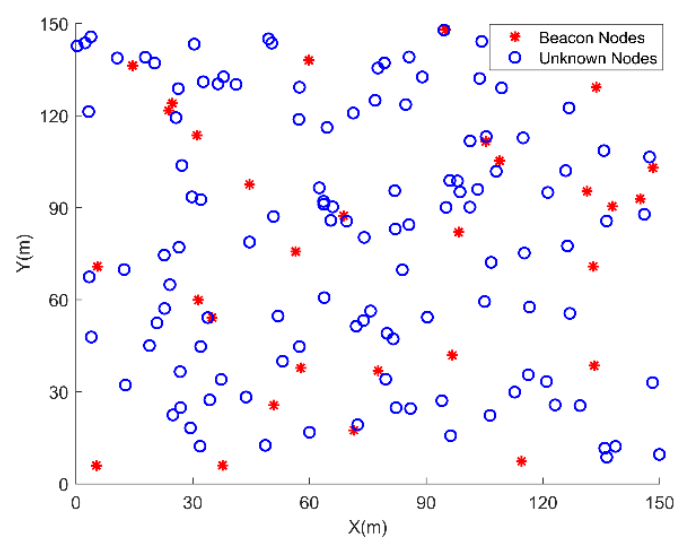

Fig 4. A Typical Example of Node Distribution in 2D Space.

\section{A. Experimental Environment}

In verifying the performance of WND-DV-Hop, comprehensive experiments were conducted in MATLAB 2016a. The experimental outcomes were compared with DVHop [10], IDV-Hop [15], CheckOut-DV-Hop [18], and NewIDV-Hop [32] in simulated settings. Table I tabulates the simulation parameters.

TABLE I. SimUlation PARAMETERS SETting

\begin{tabular}{|l|l|}
\hline Parameters & Value \\
\hline Network Size & $100 \mathrm{~m} \times 100 \mathrm{~m}$ \\
\hline Total nodes & 100 \\
\hline Beacon Nodes & 30 \\
\hline Communication Range(m) & 25 \\
\hline
\end{tabular}

\section{B. Evaluation Criteria}

In evaluating and analysing the achievement of the proposed WND-DV-Hop, localization accuracy, stability, and cost metrics had been considered.

- Accuracy Metrics

1) Localization Error (LE)

The LE refers to error between actual and calculated coordinates of unknown nodes, as defined by Equation (21).

$L E=\sqrt{\left(x_{u}-x_{a}\right)^{2}+\left(y_{u}-y_{a}\right)^{2}}$

2) Average Localization Error (ALE)

The ALE denotes the sum of localization error for unknown nodes. Its mathematical expression is as follows:
$A L E=\frac{\sum_{u, a=1}^{n} \sqrt{\left(x_{u}-x_{a}\right)^{2}+\left(y_{u}-y_{a}\right)^{2}}}{n}$

- Stability Metrics

1) Localization Error Variance (LEV)

The LEV determines the stability of localization algorithm, given as follows:

$L E V=\sqrt{\frac{\sum_{u, a=1}^{n}\left(\sqrt{\left(x_{u}-x_{a}\right)^{2}+\left(y_{u}-y_{a}\right)^{2}}-L E R \times R\right)^{2}}{n \times R^{2}}}$

Here, $n$ signifies the number of unknown nodes, whereas $\left(x_{u}, y_{u}\right)$ and $\left(x_{a}, y_{a}\right)$ are the estimated and actual coordinates of unknown nodes, respectively. $\mathrm{R}$ denotes communication range.

- Cost Metrics

1) Computational Cost

Localization time (LT) is employed to evaluate computational cost, which is measured by the time taken to locate an unknown node.

\section{Experimental Results}

Simulations for all algorithms were performed as many as 100 times for each result to assess the performance for random deployment. Abbreviations LE, ALE, LEV, and LT are used to represent localization error, average localization error, localization error variance, and localization time, respectively.

\section{- LE for Each Unknown Node}

The simulation was performed under the scenario that 100 sensor nodes were irregularly deployed in the area of $100 \times$ $100 \mathrm{~m}^{2}$ with $30 \%$ beacon node. The communication range was $25 \mathrm{~m}$.

Fig. 5 presents the LE for each unknown node under five algorithms. Apparently, the proposed algorithm (WND-DVHop) gave the best outcomes with reduction in localization error at 55\%, 35\%, 20\%, and 25\%, respectively, compared with DV-Hop [10], IDV-Hop [15], CheckOut-DV-Hop [18], and New-IDV-Hop [32].

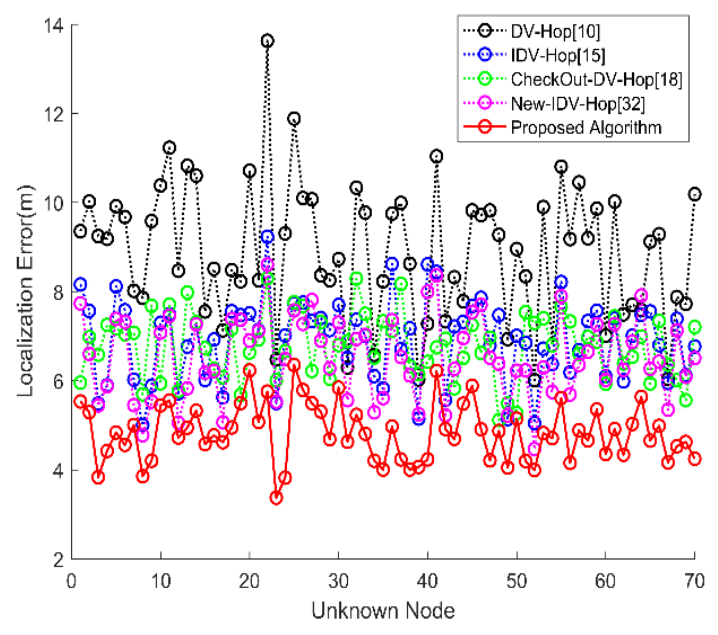

Fig 5. The LE for Each Unknown Node. 
TABLE II. LOCALIZATION ERROR AND STANDARD DEVIATION COMPARISONS

\begin{tabular}{|l|l|l|l|l|}
\hline Localization Algorithm & $\begin{array}{l}\text { Max. } \\
\text { LE(m) }\end{array}$ & $\begin{array}{l}\text { Min. } \\
\text { LE(m) }\end{array}$ & $\begin{array}{l}\text { Avg. } \\
\text { LE(m) }\end{array}$ & $\begin{array}{l}\text { Std. } \\
\text { (LE) }\end{array}$ \\
\hline DV-Hop [10] & 13.6290 & 6.0095 & 8.8912 & 1.5096 \\
\hline IDV-Hop [15] & 9.2288 & 5.0009 & 6.9184 & 1.0293 \\
\hline CheckOut-DV-Hop [18] & 8.2908 & 5.1165 & 6.9184 & 0.7299 \\
\hline New-DV-Hop [32] & 8.6 .43 & 4.4614 & 6.5341 & 1.0991 \\
\hline Proposed algorithm & $\mathbf{6 . 3 5 1 7}$ & $\mathbf{3 . 2 8 5 4}$ & $\mathbf{4 . 8 3 0 9}$ & $\mathbf{0 . 6 4 0 8}$ \\
\hline
\end{tabular}

Table II presents the LE and its standard deviation for the five localization algorithms. Upon comparing with the other four localization algorithms, the proposed algorithm yielded the lowest location error in terms of max, min, and average values. The proposed algorithm also recorded the lowest standard deviation, which indicated that WND-DV-Hop had more stability. The proposed algorithm appeared to perform better under average location error with almost 40.95\% decrease, when compared with DV-Hop [10].

- Accuracy and Stability Metrics with Variation Factors

Accuracy and stability are two of most critical factors for any algorithm. In this study, ALE and LEV were applied to determine accuracy and stability of the proposed algorithm under the effects of total number of nodes, beacon node ratio, and communication range.

\section{1) Effect of Total Number of Nodes}

During this experiment, the sensor nodes were evenly increased from 50 to 350, while communication radius and the proportion of beacon nodes ratio were fixed at $25 \mathrm{~m}$ and $10 \%$, respectively. Fig. 6, Fig. 7, Table III and Table IV list the empirical outcomes of ALE and LEV.

In Fig. 6, ALE displayed a declining trend with increment in sensor nodes. This pattern is attributable to the increasing number of nodes within the communication radius that enhanced both hop size and network connectivity. Fig. 6 shows that the proposed algorithm performed better than the other four algorithms. Its average localization error decreased to $70 \%$, 55\%, 50\%, and 53\%, when compared with the algorithm prescribed in [10,15,18,32], respectively.

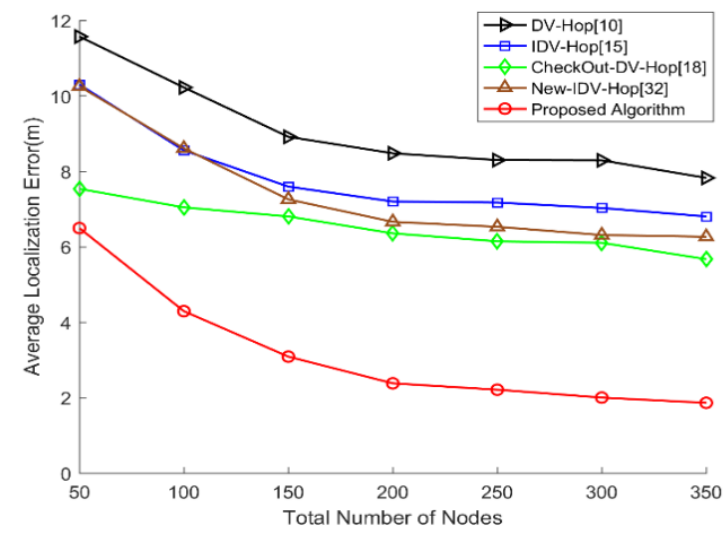

Fig 6. Simulation Results ALE Under Various Total Number of Nodes.

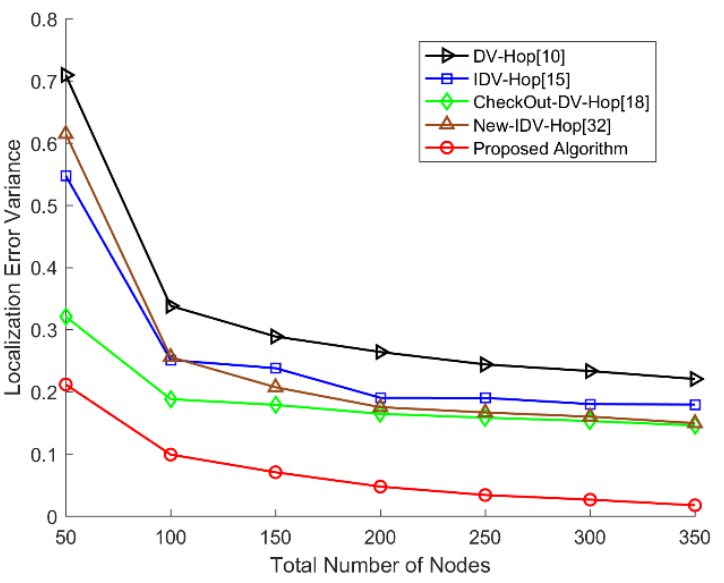

Fig 7. Simulation Results LEV Under Various Total Number of Nodes.

As portrayed in Fig. 7, reduction in LEV was noted with increment in the number of nodes in WSN. Generally, the stability of the proposed algorithm was significantly better than the rest of algorithm. In fact, the proposed algorithm displayed strong stability when sensor nodes exceeded 150. Among all algorithms, the New-IDV-Hop [32] also exerted good performance when the nodes exceeded 100.

As tabulated in Table III, the ALE of the proposed algorithm gave better performance in terms of max, min, and average values. Put simply, upon comparing with the primary DV-Hop [10], a decrease of $64.89 \%$ was noted in average term. Both IDV-Hop [15] and New-IDV-Hop [32] displayed superior performance in minimizing localization error.

TABLE III. COMPARISON ALE UNDER VARIOUS TOTAL NUMBER OF NODES

\begin{tabular}{|l|l|l|l|}
\hline \multirow{2}{*}{ Localization Algorithm } & \multicolumn{3}{|l|}{ Average Localization Error (ALE) } \\
\cline { 2 - 4 } & Max. & Min. & Avg. \\
\hline DV-Hop [10] & 11.5730 & 7.8253 & 9.0863 \\
\hline IDV-Hop [15] & 10.2991 & 6.8037 & 7.8076 \\
\hline CheckOut-DV-Hop [18] & 7.5354 & 5.6701 & 6.5226 \\
\hline New-DV-Hop [32] & 10.2543 & 6.2659 & 7.4120 \\
\hline Proposed Algorithm & $\mathbf{6 . 4 9 5 2}$ & $\mathbf{1 . 8 6 3 0}$ & $\mathbf{3 . 1 9 0 4}$ \\
\hline
\end{tabular}

TABLE IV. COMPARISON LEV UNDER VARIOUS TOTAL NUMBER OF NODES

\begin{tabular}{|l|l|l|l|}
\hline \multirow{2}{*}{ Localization Algorithm } & \multicolumn{3}{|l|}{ Localization Error Variance (LEV) } \\
\cline { 2 - 4 } & Max. & Min. & Avg. \\
\hline DV-Hop [10] & 0.7091 & 0.2205 & 0.3282 \\
\hline IDV-Hop [15] & 0.5472 & 0.1793 & 0.2537 \\
\hline CheckOut-DV-Hop [18] & 0.3207 & 0.1456 & 0.1870 \\
\hline New-DV-Hop [32] & 0.4856 & 0.2512 & 0.3126 \\
\hline Proposed Algorithm & $\mathbf{0 . 2 1 1 3}$ & $\mathbf{0 . 0 1 7 4}$ & $\mathbf{0 . 0 7 2 3}$ \\
\hline
\end{tabular}


Table IV shows that the stability performance of improved WND-DV-Hop emerged as the best amidst all algorithms; when compared with DV-HOP [10], IDV-Hop [15], CheckOut-DV-Hop [18], and New-DV-Hop [32], reduction of $77.98 \%, 71.50 \%, 61.34 \%$, and $76.87 \%$ had been recorded in average term, respectively. This is especially true when the proposed algorithm achieved the lowest location error variance in terms of max, min, and average values.

\section{2) Effect of Beacon Node Ratio}

In the experiment, the beacon ration was increased from $10 \%$ to $40 \%$, while number of nodes and communication range were fixed at 100 and $25 \mathrm{~m}$, respectively. Fig. 8, Fig. 9, Table V and Table VI present the empirical finding of ALE and LEV under various beacon node ratio.

As illustrated in Fig. 8, increment in beacon nodes ratio led to a declining trend for ALE, stemming from the reduced hop count between the nodes. Increment in beacon nodes led to hop size being approximated to the actual value. The proposed algorithm always gave the lowest values; reduction of 50, 40, 35, and 35\% when compared to DV-Hop [10], IDV-Hop [15], CheckOut-DV-Hop [18], and New-IDV-Hop [32], respectively.

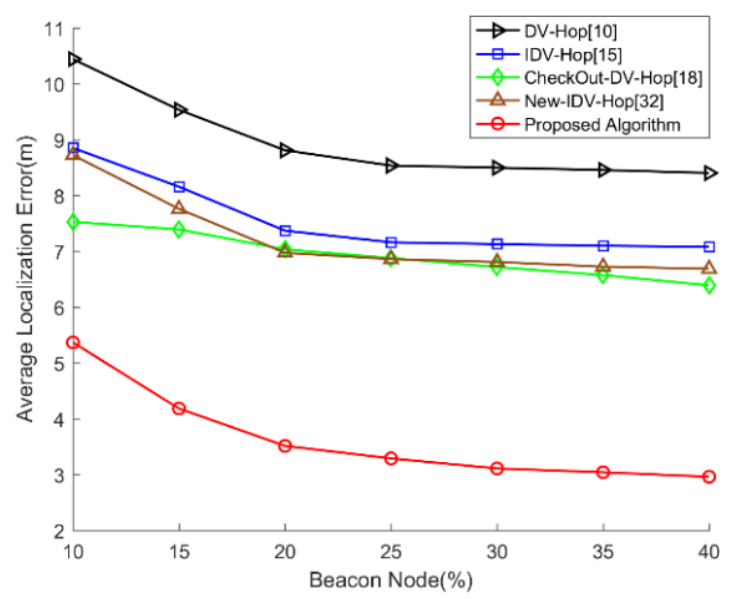

Fig 8. Simulation Results ALE Under Various Beacon Node Ratio.

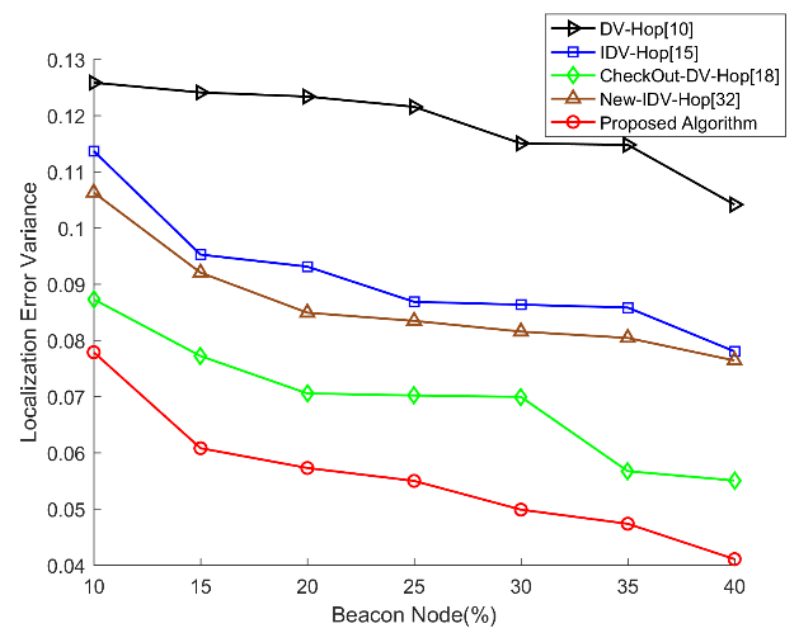

Fig 9. Simulation Results LEV Under Various Beacon Node Ratio.
TABLE V. COMPARISON ALE UNDER VARIOUS BEACON NODE RATIO

\begin{tabular}{|l|l|l|l|}
\hline \multirow{2}{*}{ Localization Algorithm } & \multicolumn{3}{|l|}{ Average Localization Error (ALE) } \\
\cline { 2 - 4 } & Max. & Min. & Avg. \\
\hline DV-Hop [10] & 10.4424 & 8.4023 & 8.9537 \\
\hline IDV-Hop [15] & 8.8509 & 7.0817 & 7.5495 \\
\hline CheckOut-DV-Hop [18] & 7.5527 & 6.3893 & 6.9306 \\
\hline New-DV-Hop [32] & 8.7218 & 6.6879 & 7.2195 \\
\hline Proposed Algorithm & $\mathbf{5 . 3 6 7 5}$ & $\mathbf{2 . 9 6 2 1}$ & $\mathbf{3 . 6 3 8 7}$ \\
\hline
\end{tabular}

TABLE VI. COMPARISON LEV UNDER VARIOUS BEACON NODE RATIO

\begin{tabular}{|l|l|l|l|}
\hline \multirow{2}{*}{ Localization Algorithm } & \multicolumn{3}{|c|}{ Localization Error Variance (LEV) } \\
\cline { 2 - 4 } & Max. & Min. & Avg. \\
\hline DV-Hop [10] & 0.1258 & 0.1041 & 0.1184 \\
\hline IDV-Hop [15] & 0.1137 & 0.0780 & 0.0913 \\
\hline CheckOut-DV-Hop [18] & 0.0873 & 0.0550 & 0.0695 \\
\hline New-DV-Hop [32] & 0.1063 & 0.0764 & 0.0864 \\
\hline Proposed Algorithm & $\mathbf{0 . 0 7 7 9}$ & $\mathbf{0 . 0 4 1 0}$ & $\mathbf{0 . 0 5 5 6}$ \\
\hline
\end{tabular}

As illustrated in Fig. 9, DV-Hop [10] exhibited the worst performance amidst the five algorithms. The proposed algorithm significantly outperformed IDV-Hop [15], and NewIDV-Hop [32]. The Checkout-DV-Hop [32] displayed better improvement, but not as exceptional as the proposed algorithm.

Table $\mathrm{V}$ shows that the ALE performance of the original DV-Hop is always the worst, while the proposed algorithm is the best. When compared with DV-Hop [10], IDV-Hop [15], CheckOut-DV-Hop [18], and New-DV-Hop [32], the average localization error deceased under average term by up to $59.36 \%$, 51.80\%, 47.50\%, and 49.60\%, respectively.

As given in Table VI, the LEV for the proposed algorithm emerged as outstanding due to the lowest max, min, and average values. The original DV-Hop is always the poorest from the rest. The LEV of the proposed algorithm decreased to $53.04 \%$, 39.10\%, 20.00\%, and 35.65\%, respectively, when compared with DV-Hop [10], IDV-Hop [15], CheckOut-DVHop [18], and New-DV-Hop [32].

\section{3) Effect of Communication Range}

In this experiment, the communication range was increased from 20 to $36 \mathrm{~m}$, while sensor and beacon nodes were fixed at 100 and 20, respectively. Fig. 10, Fig. 11, Table VII and Table VIII tabulate the empirical outcomes with varied communication ranges.

Fig. 10 illustrates five algorithms of ALE under varied communication ranges. The empirical findings show that the ALE exerted a declining trend with increment in communication range. The proposed algorithm always had the lowest localization error, which decreased about 50, 35, 30, and 25\%, respectively, when compared with DV-Hop [10], IDV-Hop [15], CheckOut-DV-Hop [32], and New-IDV-Hop [32]. Besides, ALE demonstrated only a slight change when the communication range exceeded $32 \mathrm{~m}$. 
(IJACSA) International Journal of Advanced Computer Science and Applications, Vol. 11, No. 5, 2020

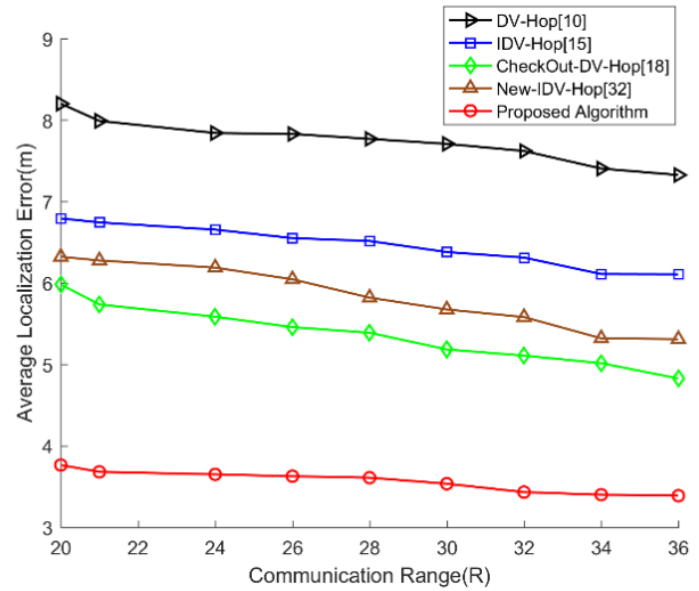

Fig 10. Simulation Results ALE Under Various Communication Range.

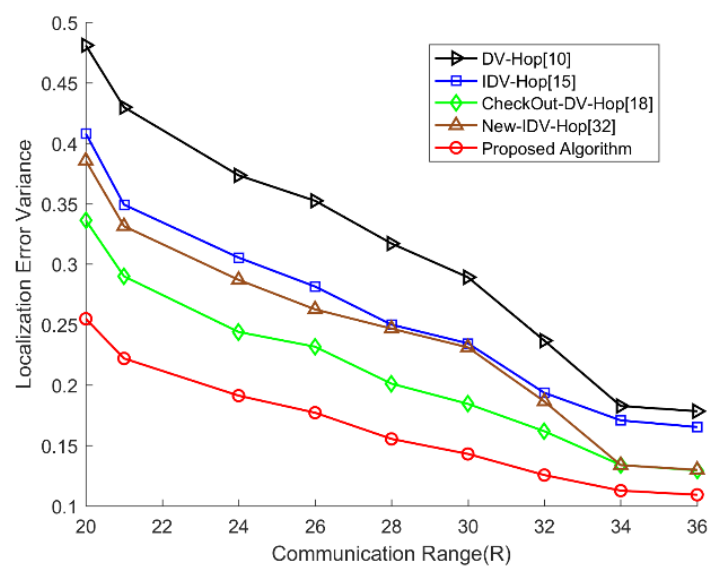

Fig 11. Simulation Results LEV Under Various Communication Range.

Increment in communication range decreased the LEV (see Fig. 11). This result is attributable to the network that is wellconnected with the increasing communication range. The proposed localization algorithm always gave the greatest performance, and was followed by CheckOut-DV-Hop [18]. The performances of DV-Hop [10], IDV-Hop [15], and NewIDV-Hop [32] were close. When the communication range exceeded $32 \mathrm{~m}$, the localization error variance for all the algorithms gave low values.

TABLE VII. COMPARISON ALE UNDER VARIOUS COMMUNICATION RANGE

\begin{tabular}{|l|l|l|l|}
\hline \multirow{2}{*}{ Localization Algorithm } & \multicolumn{3}{|l|}{ Average Localization Error (ALE) } \\
\cline { 2 - 4 } & Max. & Min. & Avg. \\
\hline DV-Hop [10] & 8.2024 & 7.3263 & 7.7440 \\
\hline IDV-Hop [15] & 6.7933 & 6.1074 & 6.4633 \\
\hline CheckOut-DV-Hop [18] & 5.9815 & 4.8280 & 5.3657 \\
\hline New-DV-Hop [32] & 6.3221 & 5.3111 & 5.8381 \\
\hline Proposed Algorithm & $\mathbf{3 . 7 6 4 8}$ & $\mathbf{3 . 3 8 8 0}$ & $\mathbf{3 . 5 6 5 4}$ \\
\hline
\end{tabular}

TABLE VIII. COMPARISON LEV UNDER VARIOUS COMMUNICATION RANGE

\begin{tabular}{|l|l|l|l|}
\hline \multirow{2}{*}{ Localization Algorithm } & \multicolumn{3}{|c|}{ Localization Error Variance (LEV) } \\
\cline { 2 - 4 } & Max. & Min. & Avg. \\
\hline DV-Hop [10] & 0.4810 & 0.1784 & 0.3156 \\
\hline IDV-Hop [15] & 0.4081 & 0.1653 & 0.2620 \\
\hline CheckOut-DV-Hop [18] & 0.3363 & 0.1294 & 0.2125 \\
\hline New-DV-Hop [32] & 0.3856 & 0.1298 & 0.2438 \\
\hline Proposed Algorithm & $\mathbf{0 . 2 5 4 8}$ & $\mathbf{0 . 1 0 9 2}$ & $\mathbf{0 . 1 6 5 7}$ \\
\hline
\end{tabular}

The result of both ALE is tabulated in Table VII. It seems that the enhanced algorithm outperformed the others for average localization error. The CheckOut-DV-Hop [32] gave the second-best performance. The average localization error of the proposed algorithm decreased to $53.96 \%, 44.84 \%$, $33.55 \%$, and $38.93 \%$ under average term, respectively, when compared with DV-Hop [10], IDV-Hop [15], CheckOut-DV-Hop [18], and New-DV-Hop [32].

Table VIII shows that the performance of proposed algorithm was outstanding in terms of max, min, and average LEV values. Upon comparing with the proposed algorithm, the best min gap of max and the best average location error variance were displayed by CheckOut-DV-Hop [18] and NewDV-Hop [32]. The original DV-Hop, on the contrary, gave the worst performance.

\section{- Cost Metrics}

In order to verify the proposed algorithm under cost metrics, the LT was calculated. This simulation experiment employed 100 nodes that were irregularly deployed in area of $100 \times 100$ $\mathrm{m}^{2}$, the beacon node ratio of $30 \%$, and communication range set at $25 \mathrm{~m}$.

Table IX presents the LT of five algorithms with 100 nodes and 30 beacon nodes. It can be concluded that the LT of the basic DV-Hop [10] algorithm was at minimum, whereas IDVHop [15] and New-DV-Hop [32] were close. The localization time of the proposed algorithm was slower than the above three algorithms, but faster than the CheckOut-DV-Hop [18] to some extent. This is ascribed to correct hop and hop count cost relative time. Hence, a pressing need is present to balance the decreasing location accuracy and localization time.

TABLE IX. COMPARISON OF ALGORITHMS WITH LOCALIZATION TIME

\begin{tabular}{|l|l|}
\hline Localization Algorithm & Localization Time(s) \\
\hline DV-Hop [10] & 0.6572 \\
\hline IDV-Hop [15] & 0.6605 \\
\hline CheckOut-DV-Hop [18] & 0.7247 \\
\hline New-DV-Hop [32] & 0.6734 \\
\hline Proposed Algorithm & 0.7112 \\
\hline
\end{tabular}




\section{CONCLUSION}

Aimed at enhancing localization accuracy, stability, and cost; an enhanced DV-Hop algorithm based on weighted factor is described in this paper. The RSSI scheme was embedded to correct one hop-count of unknown node, which transformed the one hop-count from discrete to continuous values. In order to reduce localization errors, the hop size was modified by using weighted correction factor. The estimated distance between beacon and located node was calculated by weighted hop count, instead of the nearest one. A novel weighted least squares method is proposed to address nonlinear equations in estimating node coordinates. Several parameters were weighed in to determine their effects, including communication range, total number nodes, and beacon nodes ratio. The simulation outcomes revealed that the proposed algorithm was exceptional for localization accuracy and stability, when compared with DV Hop, IDV-Hop, CheckOut-DV-Hop, and New-IDV Hop. In future, we are planning to implement WND-DV-Hop in irregular and sparse network under consider irregular radio patterns. In addition, our proposed algorithm will be assessed under much complicated three-dimensional (3D) wireless network environments with consider specific environmental factors.

\section{ACKNOWLEDGMENT}

This research was supported by Universiti Malaysia Pahang Postgraduate Research Grants, grant number PGRS1903143.

\section{REFERENCES}

[1] Akyildiz, I. F.; Su, W.; Sankarasubramaniam, Y.; Cayirci, E. J. I. C. m., A survey on sensor networks. 2002, 40, (8), 102-114.

[2] Yick, J.; Mukherjee, B.; Ghosal, D. J. C. n., Wireless sensor network survey. 2008, 52, (12), 2292-2330.

[3] Chandanapalli, S. B.; Reddy, E. S.; Lakshmi, D. R. J. I. J. o. M. L.; Cybernetics, DFTDT: distributed functional tangent decision tree for aqua status prediction in wireless sensor networks. 2018, 9, (9), 1419-1434.

[4] Suo, H.; Wan, J.; Huang, L.; Zou, C. In Issues and challenges of wireless sensor networks localization in emerging applications, 2012 International Conference on Computer Science and Electronics Engineering, 2012; IEEE: 2012; pp 447-451.

[5] Chowdhury, T. J.; Elkin, C.; Devabhaktuni, V.; Rawat, D. B.; Oluoch, J. J. C. N., Advances on localization techniques for wireless sensor networks: A survey. 2016, 110, 284-305.

[6] Laoudias, C.; Moreira, A.; Kim, S.; Lee, S.; Wirola, L.; Fischione, C. J. I. C. S.; Tutorials, A survey of enabling technologies for network localization, tracking, and navigation. 2018, 20, (4), 3607-3644.

[7] Khelifi, M.; Moussaoui, S.; Silmi, S.; Benyahia, I. J. I. J. o. S. N., Localization algorithms for wireless sensor networks: a review. 2015, 19, (2), 114-129.

[8] Nagpal, R., Organizing a global coordinate system from local information on an amorphous computer. 1999.

[9] 12.Čapkun, S.; Hamdi, M.; Hubaux, J.-P. J. C. C., GPS-free positioning in mobile ad hoc networks. 2002, 5, (2), 157-167.

[10] Niculescu, D.; Nath, B. In Ad hoc positioning system (APS), GLOBECOM'01. IEEE Global Telecommunications Conference (Cat. No. 01CH37270), 2001; IEEE: 2001; pp 2926-2931.

[11] He, T.; Huang, C.; Blum, B. M.; Stankovic, J. A.; Abdelzaher, T. In Range-free localization schemes for large scale sensor networks, Proceedings of the 9th annual international conference on Mobile computing and networking, 2003; ACM: 2003; pp 81-95.

[12] Girod, L.; Bychkovskiy, V.; Elson, J.; Estrin, D. In Locating tiny sensors in time and space: A case study, Proceedings. IEEE International
Conference on Computer Design: VLSI in Computers and Processors, 2002; IEEE: 2002; pp 214-219.

[13] Harter, A.; Hopper, A.; Steggles, P.; Ward, A.; Webster, P. J. W. N., The anatomy of a context-aware application. 2002, 8, (2/3), 187-197.

[14] Niculescu, D.; Nath, B. In Ad hoc positioning system (APS) using AOA, IEEE INFOCOM 2003. Twenty-second Annual Joint Conference of the IEEE Computer and Communications Societies (IEEE Cat. No. 03CH37428), 2003; Ieee: 2003; pp 1734-1743.

[15] Chen, K.; Wang, Z.-h.; Lin, M.; Yu, M., An improved DV-Hop localization algorithm for wireless sensor networks. 2010.

[16] Cheikhrouhou, O.; M Bhatti, G.; Alroobaea, R. J. S., A hybrid DV-hop algorithm using RSSI for localization in large-scale wireless sensor networks. 2018, 18, (5), 1469.

[17] Peng, B.; Li, L. J. C. N., An improved localization algorithm based on genetic algorithm in wireless sensor networks. 2015, 9, (2), 249-256.

[18] Gui, L.; Val, T.; Wei, A.; Dalce, R. J. A. H. N., Improvement of rangefree localization technology by a novel DV-hop protocol in wireless sensor networks. 2015, 24, 55-73.

[19] Tomic, S.; Mezei, I. J. T. S., Improvements of DV-Hop localization algorithm for wireless sensor networks. 2016, 61, (1), 93-106.

[20] Tao, Q.; Zhang, L. In Enhancement of DV-Hop by weighted hop distance, 2016 IEEE Advanced Information Management, Communicates, Electronic and Automation Control Conference (IMCEC), 2016; IEEE: 2016; pp 1577-1580.

[21] Ma, X.; Liu, W.; Wang, Z. In Node localization of wireless sensor network based on secondary correction error, International symposium on parallel architecture, algorithm and programming, 2017; Springer: 2017; pp 142-151.

[22] Song, G.; Tam, D. J. I. J. o. D. S. N., Two novel DV-Hop localization algorithms for randomly deployed wireless sensor networks. 2015, 11, (7), 187670.

[23] Zhang, B.; Ji, M.; Shan, L. In A weighted centroid localization algorithm based on DV-hop for wireless sensor network, 2012 8th International Conference on Wireless Communications, Networking and Mobile Computing, 2012; IEEE: 2012; pp 1-5.

[24] Fang, X. J. J. o. s. e., Improved DV-Hop positioning algorithm based on compensation coefficient. 2015, 9, (3), 650-657.

[25] Shahzad, F.; Sheltami, T. R.; Shakshuki, E. M. J. I. T. o. M. C., DVmaxHop: A fast and accurate range-free localization algorithm for anisotropic wireless networks. 2016, 16, (9), 2494-2505.

[26] Wang, F.; Wang, C.; Wang, Z.; Zhang, X.-y. J. I. J. o. D. S. N., A hybrid algorithm of GA+ simplex method in the WSN localization. 2015, 11, (7), 731894.

[27] Kumar, S.; Lobiyal, D. J. T. S., Novel DV-Hop localization algorithm for wireless sensor networks. 2017, 64, (3), 509-524.

[28] Kaur, A.; Kumar, P.; Gupta, G. P. J. W. P. C., Nature inspired algorithmbased improved variants of DV-Hop algorithm for randomly deployed 2D and 3D wireless sensor networks. 2018, 101, (1), 567-582.

[29] Kaur, A.; Kumar, P.; Gupta, G. P. J. W. N., A new localization using single mobile anchor and mesh-based path planning models. 2019, 25, (5), 2919-2929.

[30] Kaur, A.; Kumar, P.; Gupta, G. P., Sensor Nodes Localization for 3D Wireless Sensor Networks Using Gauss-Newton Method. In Smart Innovations in Communication and Computational Sciences, Springer: 2019; pp 187-198.

[31] Kerdabadi, M. S.; Nejad, F. P.; Ghazizadeh, R.; Farrokhi, H. J. I. J. o. U. W. C.; Systems, Wireless sensor network localization using new heuristic optimisation algorithms. 2018, 3, (4), 209-218.

[32] Prashar, D.; Jyoti, K. J. W. P. C., Distance Error Correction Based Hop Localization Algorithm for Wireless Sensor Network. 2019, 106, (3), 1465-1488

[33] Yan, X.; Sun, L.; Zhou J; Song, A., DV-hop localisation algorithm based on optimal weighted least square in irregular areas. 2018, 54, (21), 12431245. 